\title{
Using focus groups to identify the barriers of arthritis-related disability on food behaviours and guide future nutrition interventions
}

R. Bennett ${ }^{1}$, T. Demmers ${ }^{2}$, H. Plourde ${ }^{1}$, K. Arrey ${ }^{3}$, B. Armour ${ }^{4}$, G. Ferland ${ }^{5}$, L. Kakinami²

${ }^{1}$ McGill University, Montreal, QC, ${ }^{2}$ Concordia University, Montreal, QC, ${ }^{3}$ Kim Arrey Nutrition, Montreal QC, ${ }^{4}$ Dietitians

of Canada: Practice Evidence Based Nutrition (PEN), Montreal, QC, ${ }^{5}$ Université de Montreal, Montreal, QC

蚛 McGill

\section{Background and Objectives}

Background:

Arthritis is a leading cause of disability worldwide.

The association between nutrition and arthritis is an emerging topic; current research focuses on whether certain foods or diets can promote favourable clinical outcomes. Understanding how arthritis affects nutrition (food choices, grocery shopping, and food preparation) is critical to successfully adapting dietary interventions but remains largely understudied.

\section{Objectives:}

- To describe the impact of disability related to arthritis on dietary habits To gain insight on the incentives and deterrents to participating in a nutrition education program

\section{Methods}

Sampling: A convenience sample of 27 individuals with arthritis participated in one of four focus groups.

Data collection: Prior to focus group discussions, participants completed questionnaires on socioeconomic status, and disability (Stanford HAQDI). Focus groups were moderated by a dietitian and a graduate student. Audio recordings of the discussions were transcribed verbatim.

Data analysis: Data were coded using constant comparative analysis. An iterative process was used to refine and validate the coding schemes.

\begin{tabular}{l}
\multicolumn{1}{c|}{ Results } \\
\hline Sample characteristics: \\
- Mean age of 64 years (range: $35-79)$ \\
- $81 \%$ female \\
- $85.1 \%$ osteoarthritis \\
- Median annual income: $50 \mathrm{k}-75 \mathrm{k}$ \\
- Mean HAQ-DI was 0.25 (mild to \\
moderate disability)
\end{tabular}

\section{Acknowledgements}

This study was funded by the Drummond Foundation

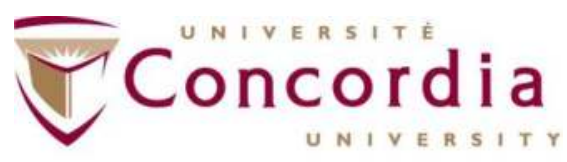

Centre PERFORM Centre
Université กิก de Montréal

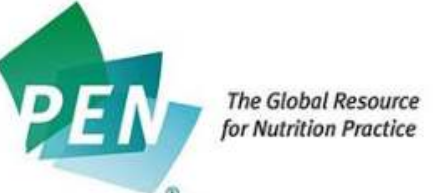

Results (cont'd)
The steps in the process leading to food consumption were reported:
(1) food choices; (2) procurement; (3) preparation; and (4) consumption.
Four main recurring themes emerged: pain; fatigue; knowledge; and social support.

Table 1. Impact of arthritis on nutrition: Summary of thematic findings

Food Choices Several did not make intentional dietary changes due to lack of awareness (knowledge)

Many reported making dietary "improvements" to alleviate their symptoms:

- $\uparrow$ fruits, vegetables, fatty fish, spices

- $\downarrow$ meat, sugar, processed foods

- Certain participants aimed for a more "balanced" diet, while others followed strict dietary rule

Sources of information included friends, family, the Internet (knowledge, social support) Some opted for pre-made, frozen or fast food during flare-ups (pain, fatigue)

Procurement Some reported no impact:

- Family members assisted with grocery shopping (social support)

Fatigue and pain were frequently mentioned barriers:

- Impact on shopping frequency (increase or decrease)

- Recourse to online grocery shopping and delivery

Grocery shopping was a valued social activity (social support)

Preparation $\quad$ Fatigue was the most frequently reported barrier. Common strategies included:

- Pre-made, frozen or fast food

- Choosing fast recipes

Pain limited the use of ingredients requiring preparation (ex: chopping):

- Some asked family members to prepare difficult ingredients (social support)

- Few used assistive devices

Strong desire to learn easy, healthy recipes (knowledge)

Consumption The act of eating was not affected in most

Portion size was affected in some:

- A few reduced portion size for weight loss

- Fatigue and pain reduced appetite in others
Pain: "I think because pain stresses you

out, for me anyway, I eat things that I

shouldn't be eating."

Knowledge: "It's hard to find reliable

information online specifically because

there's so much stuff out there that's not

evidence based."
Fatigue: "I can't live a normal life and work a normal day. I just can't. (...) If I have to put in a good-a hard day's work, l'll eat a frozen dinner because I justthere's no way. I can't do anything more." (Fatigue)

Social Support: "I just use different tools now or I have my husband peel stuff and I do the chopping, stuff like that."
Figure 2. Barriers and incentives to participating in a community-based nutrition education program Incentives

\section{Deterrents}

- Flexible timing and registration

- Low cost

- Interactive, hands-on activity

- Easy, quick, and inexpensive meals

- Take-home readings

- High activity cost

- Time-intensive

- Long lecture component

- Lack of scientific data supporting recommendations

\section{Discussion and Conclusion}

Pain and fatigue were the most frequently mentioned challenges in the steps leading to food intake. The complexity of the relationship between arthritis symptoms and diet makes it likely that a one-sizefits-all dietary approach will be insufficient.

In general, the participants' marked interest in improving their diet quality indicates that there is much need for nutritional counselling from qualified professionals. 